OPEN ACCESS

Edited by:

Roumen Kirov,

Institute of Neurobiology (BAS),

Bulgaria

Reviewed by:

Timo Partonen

National Institute for Health and

Welfare, Finland

Thorsten Mikoteit,

Psychiatric Clinics of the University of

Basel, Switzerland

Sébastien Weibel,

Hôpitaux Universitaires de Strasbourg

France

*Correspondence:

Bjørn Bjorvatn

bjorn.bjorvatn@uib.no

Specialty section:

This article was submitted to

Psychopathology,

a section of the journa

Frontiers in Psychology

Received: 16 June 2017 Accepted: 04 September 2017 Published: 20 September 2017

Citation:

Bjorvatn B, Brevik EJ, Lundervold AJ, Halmøy A, Posserud M-B, Instanes JT and Haavik J (2017) Adults with Attention Deficit Hyperactivity Disorder Report High Symptom Levels of

Troubled Sleep, Restless Legs, and Cataplexy. Front. Psychol. 8:1621. doi: 10.3389/fpsyg.2017.01621

\section{Adults with Attention Deficit Hyperactivity Disorder Report High Symptom Levels of Troubled Sleep, Restless Legs, and Cataplexy}

\author{
Bjørn Bjorvatn ${ }^{1,2 *}$, Erlend J. Brevik ${ }^{3,4,5}$, Astri J. Lundervold ${ }^{4,5}$, Anne Halmøy ${ }^{3,4}$, \\ Maj-Britt Posserud ${ }^{3,4}$, Johanne T. Instanes ${ }^{1,4}$ and Jan Haavik ${ }^{3,4}$ \\ ${ }^{1}$ Department of Global Public Health and Primary Care, University of Bergen, Bergen, Norway, ${ }^{2}$ Norwegian Competence \\ Center for Sleep Disorders, Haukeland University Hospital, Bergen, Norway, ${ }^{3}$ Division of Psychiatry, Haukeland University \\ Hospital, Bergen, Norway, ${ }^{4}$ Department of Biomedicine, K.G. Jebsen Centre for Neuropsychiatric Disorders, University of \\ Bergen, Bergen, Norway, ${ }^{5}$ Department of Biological and Medical Psychology, University of Bergen, Bergen, Norway
}

Objective: To compare the occurrence of a spectrum of different self-reported sleep problems in adults with ADHD and a control group, and to study the impact of current ADHD medication use and clinical ADHD subtype.

Method: Cross-sectional study of 268 clinically ascertained adult ADHD patients (DSM-IV criteria) and 202 randomly selected controls. Sleep problems were self-reported using validated questions, partly from Global Sleep Assessment Questionnaire.

Results: ADHD patients reported more sleep problems than controls: Lifetime occurrence of sleep problems (82.6 vs. $36.5 \%$ ), hypnotics use (61.4 vs. $20.2 \%$ ), current sleep duration below $6 \mathrm{~h}$ (26.6 vs. $7.6 \%$ ), and symptoms/signs during the past 4 weeks of excessive daytime sleepiness, cataplexy, loud snoring, breathing pauses during sleep, restless legs, and periodic limb movements in sleep (significant odds ratios ranged from 1.82 to 14.55). Current ADHD medication use was associated with less cataplexy compared with not using medication. Patients with inattentive subtype reported better sleep quality and less restless legs than patients with hyperactive/impulsive subtypes.

Conclusions: Adults with ADHD reported a very high occurrence of many different self-reported sleep problems, underlining the importance of screening for sleep disorders. Among the ADHD patients, medication use was not associated with more sleep-related symptoms, but in fact less cataplexy. When comparing ADHD subtypes, the inattentive subtype was associated with less sleep problems.

Keywords: sleep, sleepiness, snoring, apnea, restless legs, ADHD, stimulant medication, subtypes

\section{INTRODUCTION}

Attention-deficit/hyperactivity disorder (ADHD) is a common neurodevelopmental disorder. The main symptoms are inattention, hyperactivity, and impulsivity. Based on these symptoms the disorder can be categorized into three subtypes/presentations: an inattentive subtype (IA), a hyperactive/impulsive subtype (HI), and a combined subtype in which both inattentive and hyperactive/impulsive symptoms are present (American Psychiatric Association, 2000). Patients 
with ADHD also have many other symptoms, including sleep problems, which are reported to occur in $25-55 \%$ of patients with ADHD (Hvolby, 2015). In a recent systematic literature review, sleep problems were reported to be among the most common co-morbidities associated with ADHD (Instanes et al., 2016).

Poor sleep often leads to inattention/lack of concentration and mood swings, symptoms also typically seen in ADHD. Thus, it has been suggested that some patients may have been misdiagnosed with ADHD instead of a primary sleep disorder (Philipsen et al., 2006; Yoon et al., 2012; Bioulac et al., 2015). Alternatively, sleep problems may be considered an intrinsic feature of ADHD (Yoon et al., 2012; Hvolby, 2015; Hysing et al., 2016). Moreover, some patients may have co-morbid ADHD and sleep disorder (Bioulac et al., 2015). Overall, the distinction between having ADHD with sleep problems, having a sleep disorder with ADHD-like symptoms, or having co-morbid ADHD and sleep disorder is blurred and needs more systematic exploration (Oosterloo et al., 2006; Yoon et al., 2012; Bioulac et al., 2015; Hvolby, 2015).

Most studies investigating sleep problems among ADHD patients have been performed in children/adolescents (Philipsen et al., 2006; Yoon et al., 2012; Bioulac et al., 2015; Hvolby, 2015). However, it has been proposed that sleep-related problems are even more common in adults than in children with ADHD, and that the type of sleep problems may depend on age (Surman et al., 2009; Yoon et al., 2012; Hvolby, 2015).

The effects of ADHD medication on sleep are controversial (Surman and Roth, 2011; Yoon et al., 2012; Hvolby, 2015; Instanes et al., 2016). Both stimulant medication and atomoxetine have been reported to cause sleep problems as an adverse effect (Adler et al., 2009; Kirov and Brand, 2014), while other studies show that stimulants may improve sleep quality and sleep efficiency (Kooij et al., 2001; Boonstra et al., 2007; Sobanski et al., 2008). Again, most studies have been performed among children/adolescents with ADHD, and more research on the effects of ADHD medication on sleep in adults is warranted.

The relation between sleep problems and ADHD subtypes is another open question. Some studies suggest that sleep quality (Yoon et al., 2013) and daytime sleepiness (LeBourgeois et al., 2004; Chiang et al., 2010) are worse in ADHD patients with the inattentive subtype, whereas other studies suggest that sleep problems are worse in ADHD subtypes with hyperactivity/impulsivity (Corkum et al., 1999; Mayes et al., 2009; Silvestri et al., 2009). The ADHD combined subtype is characterized by an overall higher symptom severity compared with the other subtypes (Wilens et al., 2009), which may also influence the occurrence of sleep problems.

Research on the association between ADHD and sleep problems has not focused on sleep duration (Yoon et al., 2012; Hvolby, 2015; Instanes et al., 2016). However, the negative impact on health of short sleep duration, usually defined as $<6 \mathrm{~h}$ of sleep, has received increased focus (Tobaldini et al., 2017). There is evidence linking short sleep duration with increased risk of obesity, diabetes, heart disease, and mortality (Liu et al., 2017; Tobaldini et al., 2017). Thus, it is of interest to study sleep duration in ADHD patients, and the impact of ADHD medication and ADHD subtypes on sleep duration.

Sleep problems can be investigated in many different ways. Some sleep disorders require objective measurements for proper diagnosis, e.g., sleep apnea, whereas other disorders are purely based on subjective reports from the patients, e.g., restless legs syndrome (American Academy of Sleep Medicine, 2014). Most studies use self-report questionnaires. However, the quality and validity of these questionnaires differ substantially. In a recent clinical review (Klingman et al., 2017), the authors identified and evaluated different screening questionnaires for sleep disorders, and concluded that the Global Sleep Assessment Questionnaire (GSAQ) was the most suitable as a general sleep disorders screener, assessing many different sleep disorders (Roth et al., 2002).

The first aim of the present study was to compare the occurrence of different types of self-reported sleep problems, using selected and validated questions, between a large sample of adults with clinically ascertained ADHD and a randomly selected control group. We hypothesized that the ADHD patients would report more sleep problems than the controls. The second aim was to compare the occurrence of sleep problems in adult ADHD patients currently using and not using ADHD medication. The third aim was to compare the occurrence of these sleep problems in different subtypes of ADHD (IA vs. subtypes with HI).

\section{MATERIALS AND METHODS}

\section{Sample}

This cross-sectional study is part of an ongoing project on adults with ADHD in Norway (http://www.uib.no/kgjnpd). The sample comprised adults with ADHD $(n=268)$, clinically diagnosed by psychiatrists/psychologists according to the Diagnostic and Statistical Manual of Mental Disorders (DSMIV) criteria (American Psychiatric Association, 2000). The first patients were recruited from regional expert committees on $\operatorname{ADHD}(n=57)$, subsequent patients were recruited from clinical psychologists and psychiatrists in out-patient clinics nationwide $(n=211)$. The data were collected between 2011 and 2015. More details about the study can be found elsewhere (Brevik et al., 2017).

In this project we relied on detailed information provided by both participants and, for the ADHD patients, also from their treating physicians. During the course of the project, we tested two different versions of clinician-rated questionnaires; one extended version with detailed treatment details and a simplified version focusing on validation of the diagnoses (to save time for the participating clinicians). In the final sample, about half of the patients were recruited using each of these questionnaires, but there was no indication of group differences between the patients recruited by these slightly different protocols (data not shown). Thus, for half of the patients ( $n=135 / 50.4 \%$ ), we had access to clinician reported information on ADHD subtype and medication use. Of these, 54 patients belonged to the IA, 6 to the $\mathrm{HI}$, and 75 to the combined subtype. Regarding medication use, 94 patients were currently using ADHD medication (69 patients on methylphenidate, 12 on amphetamines, 3 on atomoxetine, 
7 on a combination, and 3 patients were on an unknown ADHD medication), whereas 36 patients were not using ADHD medication.

The controls $(n=202)$ were randomly selected and invited from the nation-wide Medical Birth Registry of Norway. To allow for co-morbidities and reduce selection bias, no formal exclusion criteria were used in either sample. All patients and controls were born in Norway; $90.2 \%$ of patients and $92.9 \%$ of controls stated that both parents also were of Norwegian ancestry $(p=0.4)$. All participants signed a written informed consent, and the study was approved by the Norwegian Regional Committee for Medical and Health Research Ethics, REC West [IRB \#3 (FWA00009490, IRB00001872)].

\section{Sleep Variables}

The participants completed a comprehensive sleep questionnaire (see Appendix). The questions about different sleep disorders (except the question on cataplexy) were from the GSAQ (Roth et al., 2002). To ease interpretation and readability, most questions were dichotomized in the regression analyses as "never" vs. "sometimes"/"usually"/"always" (all logistic regression analyses) or "never"/"sometimes" vs. "usually" "“always" (only for the logistic regression analyses with ADHD as dependent variable).

\section{Statistics}

Data were analyzed using SPSS v-23. Chi-square tests were used to compare categorical variables and independent samples $t$-test to compare continuous variables. Logistic regression analyses adjusted for sex and age, with ADHD as dependent variable, were used to investigate the association between different selfreported sleep problems and having ADHD. Similar adjusted logistic regression analyses were run on the clinical sample of ADHD patients (i.e., the subset of ADHD patients with detailed clinical data), with "not using ADHD medication" as the dependent variable, in order to investigate the association between "currently using" and "not using medication" on occurrence of sleep problems. Furthermore, adjusted logistic regression analyses were run on the clinical sample of ADHD patients with the inattentive ADHD subtype as the dependent variable, in order to investigate the association between the IA and the subtypes with $\mathrm{HI}$ (the HI and combined subtypes were combined due to few patients with the HI subtype) on occurrence of sleep problems. Missing values for the different sleep questions varied from 1.7 to $6.6 \%$ (breathing pauses during sleep). Only valid cases were used in the analyses. Significance levels were set at 0.05 .

\section{RESULTS}

The sex distribution between controls and ADHD patients was similar (Table 1). Mean age was 36.5 and 38.1 years among controls and ADHD patients, respectively $(p=0.06)$. Among ADHD patients, $82.6 \%$ reported to have had sleep problems (in general) that lasted 1 month or longer, compared to $36.5 \%$ among the controls (Table 1). Having used hypnotics were reported by $61.4 \%$ compared to $20.2 \%$ in the ADHD and control groups, respectively. Self-reported sleep duration in the ADHD group
TABLE 1 | Self-reported sleep problems in Norwegian adults with clinically ascertained $\operatorname{ADHD}(n=268)$ compared to a representative control group $(n=202)$.

\begin{tabular}{|c|c|c|c|c|}
\hline \multirow{2}{*}{$\begin{array}{l}\text { Characteristics and } \\
\text { self-reported sleep } \\
\text { problems }\end{array}$} & \multicolumn{2}{|c|}{$\%(n)$} & \multirow{2}{*}{$\begin{array}{c}\text { Chi-square } \\
\text { (df) }\end{array}$} & \multirow[t]{2}{*}{$p$-value ${ }^{a}$} \\
\hline & Controls & $\begin{array}{c}\text { ADHD } \\
\text { patients }\end{array}$ & & \\
\hline Sex & & & $0.4(1)$ & 0.55 \\
\hline Males & $37.1(75)$ & $40.3(108)$ & & \\
\hline Females & $62.9(127)$ & $59.7(160)$ & & \\
\hline Age & & & $27.7(2)$ & $<0.0001$ \\
\hline 18-29 years & $21.3(43)$ & $24.3(65)$ & & \\
\hline 30-44 years & $67.8(137)$ & $46.6(125)$ & & \\
\hline $45+$ years & $10.9(22)$ & $29.1(78)$ & & \\
\hline Ever had sleep problems? & & & $100.5(1)$ & $<0.0001$ \\
\hline Yes & $36.5(72)$ & $82.6(218)$ & & \\
\hline No & $63.5(125)$ & $17.4(46)$ & & \\
\hline Ever used hypnotics? & & & $76.2(1)$ & $<0.0001$ \\
\hline Yes & $20.2(40)$ & $61.4(162)$ & & \\
\hline No & 79.8 (158) & $38.6(102)$ & & \\
\hline Sleep quality & & & $80.1(4)$ & $<0.0001$ \\
\hline Very good & $32.3(63)$ & $10.1(26)$ & & \\
\hline Good & $47.7(93)$ & $30.6(79)$ & & \\
\hline Neither good nor bad & $13.3(26)$ & $28.7(74)$ & & \\
\hline Pretty bad & $6.2(12)$ & $25.2(65)$ & & \\
\hline Very bad & $0.5(1)$ & $5.4(14)$ & & \\
\hline Sleep duration & & & $25.5(1)$ & $<0.0001$ \\
\hline Below $6 \mathrm{~h}$ & $7.6(15)$ & $26.6(68)$ & & \\
\hline $6 \mathrm{~h}$ or more & $92.4(182)$ & $73.4(188)$ & & \\
\hline Circadian type & & & $20.7(4)$ & 0.0004 \\
\hline Definite morning type & $7.6(15)$ & $10.7(28)$ & & \\
\hline Moderate morning type & $25.3(50)$ & $18.3(48)$ & & \\
\hline Intermediate type & $21.7(43)$ & $20.6(54)$ & & \\
\hline Moderate evening type & $33.8(67)$ & $23.7(62)$ & & \\
\hline Definite evening type & $11.6(23)$ & $26.7(70)$ & & \\
\hline Excessive daytime sleepiness ${ }^{b}$ & & & $17.7(3)$ & 0.0005 \\
\hline Never & $57.1(113)$ & $42.4(112)$ & & \\
\hline Sometimes & $39.9(79)$ & $45.1(119)$ & & \\
\hline Usually & $2.5(5)$ & $10.6(28)$ & & \\
\hline Always & $0.5(1)$ & $1.9(5)$ & & \\
\hline Cataplexy ${ }^{\mathrm{b}}$ & & & $59.4(3)$ & $<0.0001$ \\
\hline Never & 95.5 (189) & $65.6(172)$ & & \\
\hline Sometimes & $4.0(8)$ & $28.6(75)$ & & \\
\hline Usually & $0.5(1)$ & $5.3(14)$ & & \\
\hline Always & $0.0(0)$ & $0.4(1)$ & & \\
\hline Loud snoring ${ }^{b}$ & & & $26.8(3)$ & $<0.0001$ \\
\hline Never & $56.9(111)$ & $35.9(92)$ & & \\
\hline Sometimes & $33.3(65)$ & $40.6(104)$ & & \\
\hline Usually & $9.2(18)$ & $17.6(45)$ & & \\
\hline Always & $0.5(1)$ & $5.9(15)$ & & \\
\hline Breathing pauses during sleep ${ }^{b}$ & & & $36.3(3)$ & $<0.0001$ \\
\hline Never & $92.9(182)$ & $70.2(170)$ & & \\
\hline Sometimes & $5.1(10)$ & $21.1(51)$ & & \\
\hline
\end{tabular}


TABLE 1 | Continued

\begin{tabular}{|c|c|c|c|c|}
\hline \multirow{2}{*}{$\begin{array}{l}\text { Characteristics and } \\
\text { self-reported sleep } \\
\text { problems }\end{array}$} & \multicolumn{2}{|c|}{$\%(n)$} & \multirow{2}{*}{$\begin{array}{l}\text { Chi-square } \\
\text { (df) }\end{array}$} & \multirow[t]{2}{*}{$p$-value ${ }^{a}$} \\
\hline & Controls & $\begin{array}{c}\text { ADHD } \\
\text { patients }\end{array}$ & & \\
\hline Usually & $2.0(4)$ & $5.4(13)$ & & \\
\hline Always & $0.0(0)$ & $3.3(8)$ & & \\
\hline Restless legs ${ }^{\mathrm{b}}$ & & & $76.3(3)$ & $<0.0001$ \\
\hline Never & $74.7(148)$ & $37.0(97)$ & & \\
\hline Sometimes & $23.2(46)$ & $39.3(103)$ & & \\
\hline Usually & $1.0(2)$ & $18.3(48)$ & & \\
\hline Always & $1.0(2)$ & $5.3(14)$ & & \\
\hline Periodic limb movements ${ }^{\mathrm{b}}$ & & & $61.8(3)$ & $<0.0001$ \\
\hline Never & $81.6(160)$ & $45.5(115)$ & & \\
\hline Sometimes & $15.3(30)$ & $41.9(106)$ & & \\
\hline Usually & $2.6(5)$ & $11.9(30)$ & & \\
\hline Always & $0.5(1)$ & $0.8(2)$ & & \\
\hline
\end{tabular}

a Pearson Chi-square, with Yates' correction for continuity when used in a $2 \times 2$ table.

${ }^{b}$ Symptoms/signs experienced during the past 4 weeks.

$d f$, degrees of freedom. Significant values are shown in bold.

was $6.5 \mathrm{~h}$ compared to $6.9 \mathrm{~h}$ among the controls $\left[t_{(451)}=3.2, p=\right.$ 0.002 ] , and $26.6 \%$ of the ADHD patients reported sleeping $<6 \mathrm{~h}$, compared to $7.6 \%$ of the controls (Table 1). ADHD patients more often reported to belong to the extremes of circadian type, with especially many patients reporting to be definite evening types compared to controls.

ADHD patients reported more symptoms/signs of specific sleep disorders compared with controls. This was evident for excessive daytime sleepiness, cataplexy, loud snoring, breathing pauses during sleep, restless legs, and periodic limb movements in sleep (Table 1, Figure 1). When adjusting for sex and age in the logistic regression analyses, having ADHD was markedly associated with the sleep disorders (defined as reporting such disorders "sometimes," "usually," or "always"), ranging from almost a two-fold increase (excessive daytime sleepiness) to an 11.5-fold increase (cataplexy) compared to controls (Table 2). The association between ADHD and the self-reported sleep disorders were 2.5- to 3-fold stronger for excessive daytime sleepiness and restless legs when the sleep disorders were reported "usually" or "always" (Table 2 ).

Within the ADHD group, patients currently using ADHD medication reported better sleep quality, less cataplexy, and less restless legs compared to patients not using ADHD medication (Table 3). In the adjusted logistic regression analyses, cataplexy $(\mathrm{OR}=2.47, \mathrm{CI}=1.10-5.53)$ remained significantly and positively associated with not using ADHD medication, whereas poor sleep quality $(\mathrm{OR}=2.37, \mathrm{CI}=0.95-5.93)$ and restless legs $(\mathrm{OR}=2.35, \mathrm{CI}=0.98-5.65)$ did not remain significantly associated with not using ADHD medication. None of the other sleep variables were significantly associated with ADHD medication status in the adjusted logistic regression analyses.

Within the ADHD group, there were few differences in the sleep variables between $\mathrm{ADHD}$ subtypes (combined+HI subtypes vs. IA subtype; Table 4). The exception was for restless

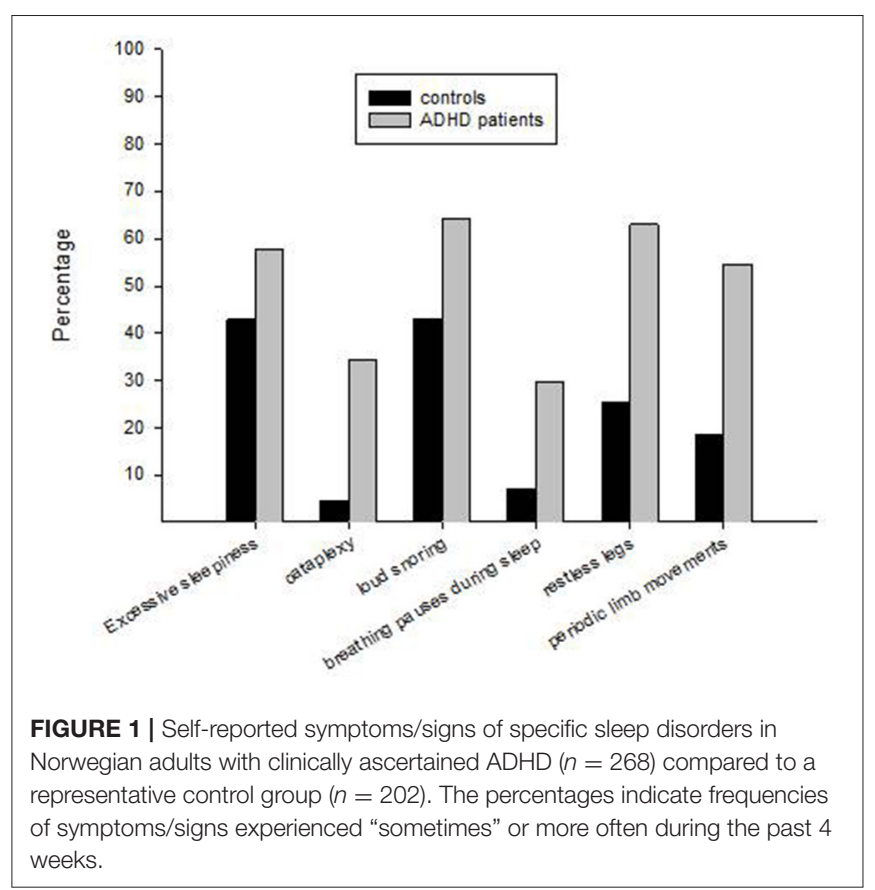

legs, with fewer patients with the inattentive subtype reporting restless legs (Table 4). This finding was confirmed in the adjusted logistic regression analysis $(\mathrm{OR}=0.29, \mathrm{CI}=0.13-0.63)$. Furthermore, sleep quality was negatively associated with the inattentive subtype $(\mathrm{OR}=0.33, \mathrm{CI}=0.15-0.73)$, indicating that patients with the combined $+\mathrm{HI}$ subtypes reported poorer sleep quality than patients with the IA subtype. None of the other sleep variables were significantly associated with ADHD subtype in the adjusted logistic regression analyses.

\section{DISCUSSION}

Our study demonstrated that a variety of specific self-reported sleep-related problems, including restless legs and cataplexy, were very common among adults with clinically ascertained ADHD. All the examined self-reported sleep variables were significantly associated with having ADHD, even when including age and sex as co-variates. Within the ADHD group, current use of ADHD medication was associated with less cataplexy. Furthermore, patients with the inattentive subtype reported less restless legs and better sleep quality compared with patients with the combined or hyperactive subtype.

There was a clear difference between ADHD patients and controls for reported sleep problems overall, with $82.6 \%$ of ADHD patients compared to $36.5 \%$ of controls having experienced a sleep problem lasting more than 1 month. In line with this, as many as $61.5 \%$ of ADHD patients reported having used hypnotics, which was three times more often than among controls. We recently published data on insomnia in this sample, showing that $66.8 \%$ of the ADHD patients compared with $28.8 \%$ of the controls fulfilled the DSM-IV criteria for insomnia disorder (Brevik et al., 2017). These findings clearly 
TABLE 2 | Logistic regression analyses with self-reported sleep variables as predictors and clinically ascertained ADHD among Norwegian adults as the dependent variable.

\begin{tabular}{cc}
\hline Adjusted OR $^{\mathrm{a}}$ & Adjusted OR $^{\mathrm{a}}$ \\
$(95 \% \mathrm{Cl})$ & $(95 \% \mathrm{Cl})$ \\
$n=438-462$ & $n=438-462$
\end{tabular}

Ever had sleep

problems?

\begin{tabular}{|c|c|}
\hline No & 1.00 \\
\hline Yes & $8.90(5.69-13.92)$ \\
\hline \multicolumn{2}{|l|}{ Ever used hypnotics? } \\
\hline No & 1.00 \\
\hline Yes & $6.47(4.20-9.97)$ \\
\hline \multicolumn{2}{|l|}{ Sleep quality } \\
\hline Good-very good & 1.00 \\
\hline Intermediate-very bad & 6.07 (3.93-9.39) \\
\hline \multicolumn{2}{|l|}{ Sleep duration } \\
\hline $6 \mathrm{~h}$ or more & 1.00 \\
\hline Below $6 \mathrm{~h}$ & $4.63(2.54-8.42)$ \\
\hline
\end{tabular}

Excessive daytime

sleepiness ${ }^{b}$

Never
Sometimes-always
Cataplexy ${ }^{b}$
Never
Sometimes-always
Loud snoring
Never
Sometimes-always
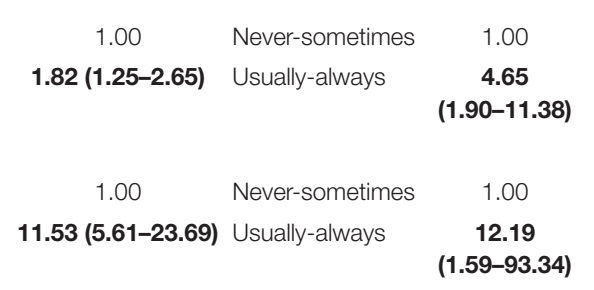

Breathing pauses during sleep $^{\mathrm{b}}$

Never
Sometimes-always

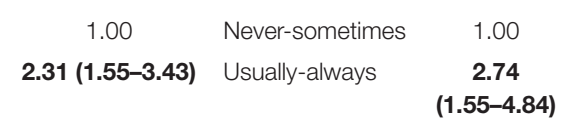

Restless legs ${ }^{\mathrm{b}}$

Never

Sometimes-always

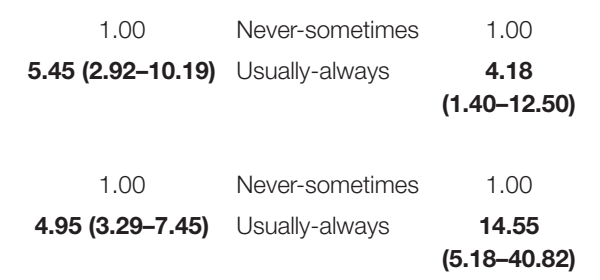

Periodic limb

movements ${ }^{b}$

Never

Sometimes-always

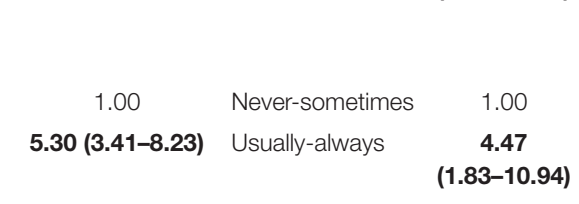

${ }^{a}$ Adjusted regression analyses with sex and age as co-variates.

${ }^{b}$ Symptoms/signs experienced during the past 4 weeks.

Significant values are shown in bold.

indicate that sleep problems are common and clinically severe among adults with ADHD, and corroborate findings from other studies (Surman et al., 2009; Fisher et al., 2014; Hvolby, 2015; Instanes et al., 2016).

To explore the association between ADHD and more specific sleep disorders, we investigated self-reported symptoms and signs of such sleep disorders during the past 4 weeks. Excessive
TABLE 3 | Comparison of self-reported sleep problems in Norwegian adults with clinically ascertained ADHD diagnosis currently using $(n=94)$ or not using $(n=36)$ ADHD medication.

\begin{tabular}{|c|c|c|c|c|}
\hline \multirow[t]{2}{*}{ Characteristics } & \multicolumn{2}{|c|}{$\%(n)$} & \multirow{2}{*}{$\begin{array}{l}\text { Chi-square } \\
\text { (df) }\end{array}$} & \multirow{2}{*}{$p$-value } \\
\hline & $\begin{array}{c}\text { ADHD } \\
\text { patients } \\
\text { using } \\
\text { medication }\end{array}$ & $\begin{array}{c}\text { ADHD } \\
\text { patients not } \\
\text { using } \\
\text { medication }\end{array}$ & & \\
\hline Sex & & & $<0.01(1)$ & 0.987 \\
\hline Males & $34.0(32)$ & $36.1(13)$ & & \\
\hline Females & $66.0(62)$ & $63.9(23)$ & & \\
\hline Age & & & $2.6(2)$ & 0.270 \\
\hline $18-29$ years & $36.2(34)$ & $22.2(8)$ & & \\
\hline 30-44 years & $48.9(46)$ & $55.6(20)$ & & \\
\hline $45+$ years & $14.9(14)$ & $22.2(8)$ & & \\
\hline Ever had sleep problems? & & & $<0.01(1)$ & 1.000 \\
\hline Yes & $85.9(79)$ & $86.1(31)$ & & \\
\hline No & $14.1(13)$ & $13.9(5)$ & & \\
\hline Ever used hypnotics? & & & $0.6(1)$ & 0.438 \\
\hline Yes & $63.0(58)$ & $71.9(23)$ & & \\
\hline No & $37.0(34)$ & $28.1(9)$ & & \\
\hline Sleep quality & & & $9.7(4)$ & 0.045 \\
\hline Very good & $13.5(12)$ & $8.6(3)$ & & \\
\hline Good & $28.1(25)$ & $14.3(5)$ & & \\
\hline Neither good nor bad & $31.5(28)$ & $28.6(10)$ & & \\
\hline Pretty bad & $22.5(20)$ & $28.6(10)$ & & \\
\hline Very bad & $4.5(4)$ & $20.0(7)$ & & \\
\hline Sleep duration & & & $1.8(1)$ & 0.183 \\
\hline Below $6 \mathrm{~h}$ & $25.8(23)$ & $40.0(14)$ & & \\
\hline $6 \mathrm{~h}$ or more & $74.2(66)$ & $60.0(21)$ & & \\
\hline Circadian type & & & $7.9(4)$ & 0.096 \\
\hline Definite morning type & $3.3(3)$ & $16.7(6)$ & & \\
\hline Moderate morning type & $19.6(18)$ & $16.7(6)$ & & \\
\hline Intermediate type & $20.7(19)$ & $22.2(8)$ & & \\
\hline Moderate evening type & $22.8(21)$ & $22.2(8)$ & & \\
\hline Definite evening type & $33.7(31)$ & $22.2(8)$ & & \\
\hline $\begin{array}{l}\text { Excessive daytime } \\
\text { sleepiness }^{b}\end{array}$ & & & $7.0(3)$ & 0.073 \\
\hline Never & $47.8(44)$ & $30.6(11)$ & & \\
\hline Sometimes & $40.2(37)$ & $44.4(16)$ & & \\
\hline Usually & $9.8(9)$ & $25.0(9)$ & & \\
\hline Always & $2.2(2)$ & $0.0(0)$ & & \\
\hline Cataplexy $^{\mathrm{b}}$ & & & $9.3(3)$ & 0.026 \\
\hline Never & $70.3(64)$ & $50.0(18)$ & & \\
\hline Sometimes & $26.4(24)$ & $36.1(13)$ & & \\
\hline Usually & $2.2(2)$ & $13.9(5)$ & & \\
\hline Always & $1.1(1)$ & $0.0(0)$ & & \\
\hline Loud snoring ${ }^{b}$ & & & $3.2(3)$ & 0.362 \\
\hline Never & $40.2(37)$ & $32.4(11)$ & & \\
\hline Sometimes & $41.3(38)$ & $41.2(14)$ & & \\
\hline Usually & $16.3(15)$ & $17.6(6)$ & & \\
\hline Always & $2.2(2)$ & $8.8(3)$ & & \\
\hline
\end{tabular}

(Continued) 
TABLE 3 | Continued

\begin{tabular}{|c|c|c|c|c|}
\hline \multirow[t]{2}{*}{ Characteristics } & \multicolumn{2}{|c|}{$\%(n)$} & \multirow{2}{*}{$\begin{array}{l}\text { Chi-square } \\
\text { (df) }\end{array}$} & \multirow{2}{*}{$p$-value ${ }^{a}$} \\
\hline & $\begin{array}{c}\text { ADHD } \\
\text { patients } \\
\text { using } \\
\text { medication }\end{array}$ & $\begin{array}{c}\text { ADHD } \\
\text { patients not } \\
\text { using } \\
\text { medication }\end{array}$ & & \\
\hline $\begin{array}{l}\text { Breathing pauses during } \\
\text { sleep } b\end{array}$ & & & $0.1(3)$ & 0.992 \\
\hline Never & $77.0(67)$ & $77.4(24)$ & & \\
\hline Sometimes & $17.2(15)$ & $16.1(5)$ & & \\
\hline Usually & $2.3(2)$ & $3.2(1)$ & & \\
\hline Always & $3.4(3)$ & $3.2(1)$ & & \\
\hline Restless legs ${ }^{b}$ & & & $13.0(3)$ & 0.005 \\
\hline Never & $44.4(40)$ & $25.0(9)$ & & \\
\hline Sometimes & $38.9(35)$ & $27.8(10)$ & & \\
\hline Usually & $14.4(13)$ & $38.9(14)$ & & \\
\hline Always & $2.2(2)$ & $8.3(3)$ & & \\
\hline Periodic limb movements ${ }^{\mathrm{b}}$ & & & $7.8(3)$ & 0.051 \\
\hline Never & $45.6(41)$ & $54.3(19)$ & & \\
\hline Sometimes & $48.9(44)$ & $28.6(10)$ & & \\
\hline Usually & $5.6(5)$ & $14.3(5)$ & & \\
\hline Always & $0.0(0)$ & $2.9(1)$ & & \\
\hline
\end{tabular}

a Pearson Chi-square, with Yates' correction for continuity when used in a $2 \times 2$ table.

${ }^{b}$ Symptoms/signs experienced during the past 4 weeks.

$d$, degrees of freedom. Significant values are shown in bold

daytime sleepiness and cataplexy are symptoms typically seen in hypersomnias (American Academy of Sleep Medicine, 2014). While excessive daytime sleepiness may also be present in other sleep disorders (e.g., sleep-related breathing disorders) and in psychiatric (e.g., depression) and somatic (e.g., hypothyroidism) disorders, cataplexy is considered a specific sign of narcolepsy type 1 (American Academy of Sleep Medicine, 2014). Narcolepsy is characterized by excessive daytime sleepiness, sleep attacks, REM sleep pathology and cataplexy (American Academy of Sleep Medicine, 2014). Both self-reported excessive daytime sleepiness and cataplexy were highly associated with ADHD in the present study, where strikingly, having ADHD was associated with a 12fold increased risk of reporting cataplexy during the past 4 weeks. Other studies have also reported high associations between ADHD and hypersomnias (Oosterloo et al., 2006; Surman et al., 2009; Ohayon, 2013; Yoon et al., 2013; Bioulac et al., 2015). Both disorders are treated with stimulant medication, suggesting a possible relation between the two conditions (Instanes et al., 2016).

Reporting symptoms or signs of restless legs and periodic limb movements in sleep (American Academy of Sleep Medicine, 2014) were also strongly associated with having ADHD in the present study. Having ADHD was associated with a 14.5 -fold increased risk of reporting restless legs "usually" or "always" during the past 4 weeks. Other studies with smaller samples also show a strong association between ADHD and restless legs/periodic limb movements in sleep (Wagner et al., 2004; Philipsen et al., 2005; Schredl et al., 2007; Zak et al., 2009). Whether some of the ADHD patients may be misdiagnosed
TABLE 4 | The influence of ADHD subtypes on self-reported sleep problems in Norwegian adults with clinically ascertained ADHD $(n=135)$.

\begin{tabular}{|c|c|c|c|c|}
\hline \multirow[t]{2}{*}{ Characteristics } & \multicolumn{2}{|c|}{ ADHD subtypes \% (n) } & \multirow{2}{*}{$\begin{array}{l}\text { Chi-square } \\
\text { (df) }\end{array}$} & \multirow[t]{2}{*}{$p$-value ${ }^{a}$} \\
\hline & Combined+HI & IA & & \\
\hline Sex & & & $2.5(1)$ & 0.115 \\
\hline Males & $29.6(24)$ & $44.4(24)$ & & \\
\hline Females & $70.4(57)$ & $55.6(30)$ & & \\
\hline Age & & & $0.4(2)$ & 0.799 \\
\hline 18-29 years & $37.0(30)$ & $31.5(17)$ & & \\
\hline 30-44 years & $48.1(39)$ & $51.9(28)$ & & \\
\hline $45+$ years & $14.8(12)$ & $16.7(9)$ & & \\
\hline Ever had sleep problems? & & & $<0.1(1)$ & 1.000 \\
\hline Yes & $87.3(69)$ & $87.0(47)$ & & \\
\hline No & $12.7(10)$ & $13.0(7)$ & & \\
\hline Ever used hypnotics? & & & $<0.1(1)$ & 1.000 \\
\hline Yes & $65.8(52)$ & $64.8(35)$ & & \\
\hline No & $34.2(27)$ & $35.2(19)$ & & \\
\hline Sleep quality & & & $8.8(4)$ & 0.066 \\
\hline Very good & $6.6(5)$ & $15.1(8)$ & & \\
\hline Good & $22.4(17)$ & 35.8 (19) & & \\
\hline Neither good nor bad & $31.6(24)$ & $22.6(12)$ & & \\
\hline Pretty bad & $25.0(19)$ & $22.6(12)$ & & \\
\hline Very bad & $14.5(11)$ & $3.8(2)$ & & \\
\hline Sleep duration & & & $1.8(1)$ & 0.182 \\
\hline Below $6 \mathrm{~h}$ & $34.7(26)$ & $22.2(12)$ & & \\
\hline $6 \mathrm{~h}$ or more & $65.3(49)$ & $77.8(42)$ & & \\
\hline Circadian type & & & $4.5(4)$ & 0.345 \\
\hline Definite morning type & $6.4(5)$ & $3.7(2)$ & & \\
\hline Moderate morning type & $16.7(13)$ & $22.2(12)$ & & \\
\hline Intermediate type & $19.2(15)$ & $20.4(11)$ & & \\
\hline Moderate evening type & $19.2(15)$ & $29.6(16)$ & & \\
\hline Definite evening type & $38.5(30)$ & $24.1(13)$ & & \\
\hline $\begin{array}{l}\text { Excessive daytime } \\
\text { sleepiness }^{b}\end{array}$ & & & $5.0(3)$ & 0.173 \\
\hline Never & $38.0(30)$ & $44.4(24)$ & & \\
\hline Sometimes & $38.0(30)$ & $46.3(25)$ & & \\
\hline Usually & $21.5(17)$ & $7.4(4)$ & & \\
\hline Always & $2.5(2)$ & $1.9(1)$ & & \\
\hline Cataplexy ${ }^{b}$ & & & $1.5(2)$ & 0.464 \\
\hline Never & $59.5(47)$ & $69.8(37)$ & & \\
\hline Sometimes & $34.2(27)$ & $26.4(14)$ & & \\
\hline Usually & $6.3(5)$ & $3.8(2)$ & & \\
\hline Always & $0.0(0)$ & $0.0(0)$ & & \\
\hline Loud snoring ${ }^{b}$ & & & $0.4(3)$ & 0.945 \\
\hline Never & $42.1(32)$ & $39.6(21)$ & & \\
\hline Sometimes & $36.8(28)$ & $37.7(20)$ & & \\
\hline Usually & $15.8(12)$ & $18.9(10)$ & & \\
\hline Always & $5.3(4)$ & $3.8(2)$ & & \\
\hline $\begin{array}{l}\text { Breathing pauses during } \\
\text { sleep }^{b}\end{array}$ & & & $0.6(3)$ & 0.904 \\
\hline Never & $79.4(54)$ & $73.6(39)$ & & \\
\hline Sometimes & $14.7(10)$ & $18.9(10)$ & & \\
\hline Usually & $2.9(2)$ & $3.8(2)$ & & \\
\hline
\end{tabular}

(Continued) 
TABLE 4 | Continued

\begin{tabular}{|c|c|c|c|c|}
\hline \multirow[t]{2}{*}{ Characteristics } & \multicolumn{2}{|c|}{ ADHD subtypes \% (n) } & \multirow{2}{*}{$\begin{array}{l}\text { Chi-square } \\
\text { (df) }\end{array}$} & \multirow[t]{2}{*}{$p$-value ${ }^{a}$} \\
\hline & Combined $+\mathrm{HI}$ & IA & & \\
\hline Always & $2.9(2)$ & $3.8(2)$ & & \\
\hline Restless legs ${ }^{\mathrm{b}}$ & & & $9.3(3)$ & 0.025 \\
\hline Never & $28.2(22)$ & $53.7(29)$ & & \\
\hline Sometimes & 42.3 (33) & 29.6 (16) & & \\
\hline Usually & $23.1(18)$ & $14.8(8)$ & & \\
\hline Always & 6.4 (5) & $1.9(1)$ & & \\
\hline Periodic limb movements ${ }^{b}$ & & & $3.2(3)$ & 0.365 \\
\hline Never & 46.7 (35) & $55.6(30)$ & & \\
\hline Sometimes & $41.3(31)$ & 40.7 (22) & & \\
\hline Usually & 10.7 (8) & $3.7(2)$ & & \\
\hline Always & $1.3(1)$ & $0.0(0)$ & & \\
\hline
\end{tabular}

a Pearson Chi-square, with Yates' correction for continuity when used in a $2 \times 2$ table. ${ }^{b}$ Symptoms/signs experienced during the past 4 weeks.

$d$, degrees of freedom. IA, Inattentive subtype. HI, Hyperactive/Impulsive subtype. Significant values are shown in bold.

or have restless legs syndrome as a co-morbid disorder is not possible to determine based on our findings.

Loud snoring and breathing pauses during sleep are symptoms/signs of obstructive sleep apnea (American Academy of Sleep Medicine, 2014). Having ADHD was associated with a 2to 3-fold higher risk of self-reported loud snoring and a 4- to 6fold higher risk of self-reported breathing pauses during sleep, dependent on the cut-off applied for having such complaints. Other studies have also found a high association between ADHD and sleep apnea (Ball et al., 1999; Surman et al., 2006; Levy et al., 2009; Youssef et al., 2011). Since both disorders may cause similar daytime symptoms, some authors have suggested that sleep apnea may be misdiagnosed as ADHD (Ball et al., 1999; Naseem et al., 2001; Bioulac et al., 2015). However, as sleep apnea can only be diagnosed by objective measures (American Academy of Sleep Medicine, 2014), we do not know whether some of our ADHD patients in fact suffer from this sleep disorder.

In addition to the strong association between ADHD and proxies for specific sleep disorders, adults with ADHD reported worse sleep quality and shorter sleep duration. About $27 \%$ of the patients reported $<6 \mathrm{~h}$ of sleep compared to $7.6 \%$ among the controls. Considering the extensive research showing that short sleep duration is linked to somatic diseases (Liu et al., 2017; Tobaldini et al., 2017), this has profound implications. Studies show that adults with ADHD have a delayed circadian rhythm (Van Veen et al., 2010; Coogan and McGowan, 2017). We did not ask specific questions about circadian rhythm sleepwake disorders, but included a question about circadian rhythm preference. In line with other studies (Van Veen et al., 2010), more adults with ADHD in the present study reported being definite evening types (26.7 vs. $11.6 \%$ ). Surprisingly, more adults with ADHD also reported being definite morning types (10.7 vs. $7.6 \%)$. By this, our data suggest that ADHD patients more often belong to the extremes of circadian type. This finding warrants further investigation.
From a clinical perspective, our findings raise concern. For instance, as many as $8.7 \%$ of the patients reported breathing pauses during sleep "usually" or "always" during the past 4 weeks. It is strongly recommended to further investigate such complaints, since breathing pauses during sleep are indicative of sleep apnea (American Academy of Sleep Medicine, 2014). Our study cannot answer whether patients or controls who reported severe sleep problems have received sleep diagnostics. However, in clinical practice symptoms like sleep problems, daytime sleepiness, and concentration problems may easily be attributed to the ADHD condition as such, and not to other potentially serious disorders. Our study therefore stresses the importance of a thorough investigation of sleep in patients with ADHD, as co-morbid sleep disorders seem to be highly prevalent.

Within the ADHD group, patients not using ADHD medication reported more cataplexy, worse sleep quality, and more restless legs. Notably, the latter two symptoms did not reach significance in the logistic regressions with adjustment for age and sex. In line with the present findings, our recent paper on insomnia in this sample showed that patients using ADHD medication reported less insomnia symptoms (Brevik et al., 2017). Thus, our data suggest that ADHD medication may improve certain sleep-related symptoms in adults with ADHD. These findings are in line with several other studies (Kooij et al., 2001; Boonstra et al., 2007; Sobanski et al., 2008; Surman and Roth, 2011), but in contrast to studies showing that such medication may impair sleep (Adler et al., 2009; Kirov and Brand, 2014).

With regards to $\mathrm{ADHD}$ subtypes, patients with the inattentive subtype reported less restless legs and better sleep quality compared to the combined+hyperactive/impulsive subtype. These findings are in contrast to studies showing that sleep quality is worse (Yoon et al., 2013) and daytime sleepiness is higher (Oosterloo et al., 2006; Chiang et al., 2010) in the inattentive subtype. However, the study by Yoon et al. (2013) used Pittsburgh Sleep Quality Index (PSQI) as the measure of sleep quality. PSQI is a nine-item questionnaire that also includes questions not directly related to self-reported quality of sleep, possibly explaining the differences between our and their study. Similar to our study, one study showed that non-medicated adults with the inattentive subtype report better sleep quality than adults with the hyperactive/impulsive subtype (Mahajan et al., 2010). Furthermore, in line with our study, several other studies suggest that sleep problems are worse in the HI subtype (Corkum et al., 1999; Mayes et al., 2009; Silvestri et al., 2009; Van Veen et al., 2010). It is however noteworthy that most sleep variables did not differ depending on ADHD subtype. This may imply that the ADHD subtype does not have a major defining role in the type of sleep problem experienced.

The present study had several strengths and limitations. The large sample with clinically ascertained adult ADHD diagnosis was an asset. Similarly, the controls were representative of the Norwegian adult population. In a representative sample of 5,000 Norwegian adults, $40.5 \%$ reported to have ever experienced a sleep problem lasting more than 1 month, and 19\% reported to have used hypnotics (Omvik et al., 2010), similar occurrences as reported among the controls in our study (36.5 and $20.2 \%$, 
respectively). This confirms that the controls recruited were representative of the general Norwegian adult population. The age and sex distributions in patients and controls were rather similar, but still, we adjusted for these variables in the logistic regression analyses. Thus, age- or sex-dependent impact on the sleep variables were controlled for. Most questions about sleep problems came from validated questionnaires or from earlier published studies, strengthening the interpretation of the results and making comparisons to other studies possible. GSAQ is recommended as the questionnaire of choice when screening for sleep disorders (Klingman et al., 2017). The questionnaire is brief and covers several different sleep-related complaints, making it especially useful in clinical practice. However, it is not a diagnostic tool since only one question per sleep disorder is included. As all sleep data were selfreported, no formal sleep disorder diagnosis can be made. Our study included questions which may be difficult for participants to respond to, e.g., loud snoring, breathing pauses during sleep and periodic limb movements. These questions rely on reports from bed partners, introducing possible response bias. Furthermore, cataplexy was much more commonly reported than expected, and we recommend caution when interpreting the frequency of this complaint. Some participants may have misinterpreted the question, but even so, the large difference between controls and patients seems valid, as all participants responded to the same wording. Multiple statistical tests were performed, without controlling for the number of tests. Thus, careful interpretation of the findings is recommended, especially when the statistical analyses showed $p$-values close to 0.05 . Moreover, the study was cross-sectional, impeding any inference about causality. Another limitation relates to medication use. This was reported by the clinician, but we do not have information about treatment duration, compliance, or whether patients currently not using ADHD medication had previously used such medication. Also, the number of ADHD patients without medication was low, making these data less robust. Some may consider it a limitation not to adjust for other comorbidities in the analyses, such as anxiety and depression. Both ADHD and sleep disorders are often co-morbid with other psychiatric and somatic disorders. We recently reported that the prevalence of anxiety/depression among our adult ADHD patients was $66.3 \%$ compared to $16.9 \%$ among the controls ( $p<0.001$; Brevik et al., 2017). Having psychiatric comorbidity will likely add to the general symptom load, and also possibly influence the associations between ADHD and sleep problems. However, in recent years it has been recognized that sleep disorders need independent clinical attention as separate disorders regardless of other conditions (American Academy of Sleep Medicine, 2014). One reason for this shift is that it is often impossible to determine whether the sleep problem is

\section{REFERENCES}

American Academy of Sleep Medicine (2014). The International Classification of Sleep Disorders, 3rd Edn. Diagnostic and Coding Manual. Darien, IL. caused by or causing, for instance, depression. Adjusting for other co-morbidities may therefore deflate the importance of the sleep problem. Furthermore, controls were recruited without exclusion criteria. This implies that some controls may have had ADHD or ADHD-like symptoms. This would reduce the chance of finding differences when comparing controls with the ADHD group. According to our IRB approved protocol, our intention was to recruit similar numbers of cases and controls. However, due to the slightly lower response rates from the controls, the final number of valid data collected from controls was lower than from the patient group. Another limitation was that we do not report data about the severity of ADHD. This makes the interpretation of medication use difficult, as untreated patients may suffer from less severe disorder, or patients not on medication may have stopped taking them because of inefficacy.

In conclusion, our study showed that adult $\mathrm{ADHD}$ patients reported considerably more of both general sleep impairment and specific sleep-related problems, including self-reported restless legs and cataplexy, compared to a representative control group. Current use of ADHD medication was not associated with worse sleep, but in fact fewer sleep-related symptoms. There were small differences in the sleep variables in relation to the ADHD subtype, except for better sleep quality and less restless legs among patients belonging to the inattentive subtype. Taken together, these findings underline the importance of screening for sleep disorders as part of the diagnostic assessment and treatment of ADHD.

\section{AUTHOR CONTRIBUTIONS}

All authors contributed substantially to the conception/design of the work, or the analysis or interpretation of the data. Furthermore, all authors drafted or revised the paper, and approved the final version, and agreed to be accountable for all aspects of the work.

\section{FUNDING}

This study was supported by the Western Norway Regional Health Authorities (Helse Vest), Stiftelsen Kristian Gerhard Jebsen, the University of Bergen, The Norwegian national research network for ADHD, and European Community's 7th Framework Program under grant agreement 631709.

\section{ACKNOWLEDGMENTS}

We wish to thank all patients and controls who volunteered to participate in this study, and Lisa Vårdal for her work with patient recruitment and data collection.

American Psychiatric Association (2000). Diagnostic and Statistical Manual of Mental Disorders. Text Revision (DSM-IV-TR). Washington, DC: American Psychiatric Association.

Adler, L. A., Liebowitz, M., Kronenberger, W., Qiao, M., Rubin, R., Hollandbeck, M., et al. (2009). Atomoxetine treatment in adults with 
attention-deficit/hyperactivity disorder and comorbid social anxiety disorder. Depress. Anxiety 26, 212-221. doi: 10.1002/da.20549

Ball, J. D., Wooten, V., and Crowell, T. (1999). Adult ADHD and/or sleep apnea? Differential diagnostic considerations with six case studies. J. Clin. Psychol. Med. Settings 6, 259-271. doi: 10.1023/A:10262397 21447

Bioulac, S., Micoulaud-Franchi, J. A., and Philip, P. (2015). Excessive daytime sleepiness in patients with ADHD-diagnostic and management strategies. Curr. Psychiatry Rep. 17:608. doi: 10.1007/s11920-015-0608-7

Boonstra, A. M., Kooij, J. J., Oosterlaan, J., Sergeant, J. A., Buitelaar, J. K., and Van Someren, E. J. (2007). Hyperactive night and day? Actigraphy studies in adult ADHD: a baseline comparison and the effect of methylphenidate. Sleep 30, 433-442. doi: 10.1093/sleep/30.4.433

Brevik, E. J., Lundervold, A. J., Halmøy, A., Posserud, M. B., Instanes, J. T., Bjorvatn, B., et al. (2017). Prevalence and clinical correlates of insomnia in adults with attention-deficit hyperactivity disorder. Acta Psychiatr. Scand. 136, 220-227. doi: 10.1111/acps.12756

Chiang, H. L., Gau, S. S., Ni, H. C., Chiu, Y. N., Shang, C. Y., Wu, Y. Y., et al. (2010). Association between symptoms and subtypes of attention-deficit hyperactivity disorder and sleep problems/disorders. J. Sleep Res. 19, 535-545. doi: $10.1111 / j .1365-2869.2010 .00832 . x$

Coogan, A. N., and McGowan, N. M. (2017). A systematic review of circadian function, chronotype and chronotherapy in attention deficit hyperactivity disorder. Atten. Defic. Hyperact. Disord. 9, 129-147. doi: 10.1007/s12402-0160214-5

Corkum, P., Moldofsky, H., Hogg-Johnson, S., Humphries, T., and Tannock, R. (1999). Sleep problems in children with attention-deficit/hyperactivity disorder: impact of subtype, comorbidity, and stimulant medication. J. Am. Acad. Child Adolesc. Psychiatry 38, 1285-1293. doi: 10.1097/00004583199910000-00018

Fisher, B. C., Garges, D. M., Yoon, S. Y., Maguire, K., Zipay, D., Gambino, M., et al. (2014). Sex differences and the interaction of age and sleep issues in neuropsychological testing performance across the lifespan in an ADD/ADHD sample from the years 1989 to 2009. Psychol. Rep. 114, 404-438. doi: 10.2466/15.10.PR0.114k23w0

Hvolby, A. (2015). Associations of sleep disturbance with ADHD: implications for treatment. Atten. Defic. Hyperact. Disord. 7, 1-18. doi: 10.1007/s12402-014-0151-0

Hysing, M., Lundervold, A. J., Posserud, M. B., and Sivertsen, B. (2016). Association between sleep problems and symptoms of attention deficit hyperactivity disorder in adolescence: results from a large populationbased study. Behav. Sleep Med. 14, 550-564. doi: 10.1080/15402002.2015.10 48448

Instanes, J. T., Klungsoyr, K., Halmoy, A., Fasmer, O. B., and Haavik, J. (2016). Adult ADHD and comorbid somatic disease: a systematic literature review. J. Atten. Disord. doi: 10.1177/1087054716669589. [Epub ahead of print].

Kirov, R., and Brand, S. (2014). Sleep problems and their effect in ADHD. Expert Rev. Neurother. 14, 287-299. doi: 10.1586/14737175.2014.885382

Klingman, K. J., Jungquist, C. R., and Perlis, M. L. (2017). Questionnaires that screen for multiple sleep disorders. Sleep Med. Rev. 32, 37-44. doi: $10.1016 /$ j.smrv.2016.02.004

Kooij, J. J., Middelkoop, H. A., Van Gils, K., and Buitelaar, J. K. (2001). The effect of stimulants on nocturnal motor activity and sleep quality in adults with ADHD: an open-label case-control study. J. Clin. Psychiatry 62, 952-956. doi: 10.4088/JCP.v62n1206

LeBourgeois, M. K., Avis, K., Mixon, M., Olmi, J., and Harsh, J. (2004). Snoring, sleep quality, and sleepiness across attention-deficit/hyperactivity disorder subtypes. Sleep 27, 520-525.

Levy, L. D., Fleming, J. P., and Klar, D. (2009). Treatment of refractory obesity in severely obese adults following management of newly diagnosed attention deficit hyperactivity disorder. Int. J. Obes. 33, 326-334. doi: 10.1038/ijo. 2009.5

Liu, T. Z., Xu, C., Rota, M., Cai, H., Zhang, C., Shi, M. J., et al. (2017). Sleep duration and risk of all-cause mortality: a flexible, non-linear, metaregression of 40 prospective cohort studies. Sleep Med. Rev. 32, 28-36. doi: 10.1016/j.smrv.2016.02.005

Mahajan, N., Hong, N., Wigal, T. L., and Gehricke, J. G. (2010). Hyperactiveimpulsive symptoms associated with self-reported sleep quality in nonmedicated adults with ADHD. J. Atten. Disord. 14, 132-137. doi: $10.1177 / 1087054709347170$

Mayes, S. D., Calhoun, S. L., Bixler, E. O., Vgontzas, A. N., Mahr, F., HillwigGarcia, J., et al. (2009). ADHD subtypes and comorbid anxiety, depression, and oppositional-defiant disorder: differences in sleep problems. J. Pediatr. Psychol. 34, 328-337. doi: 10.1093/jpepsy/jsn083

Naseem, S., Chaudhary, B., and Collop, N. (2001). Attention deficit hyperactivity disorder in adults and obstructive sleep apnea. Chest 119, 294-296. doi: 10.1378/chest.119.1.294

Ohayon, M. M. (2013). Narcolepsy is complicated by high medical and psychiatric comorbidities: a comparison with the general population. Sleep Med. 14, 488-492. doi: 10.1016/j.sleep.2013.03.002

Omvik, S., Pallesen, S., Bjorvatn, B., Sivertsen, B., Havik, O. E., and Nordhus, I. H. (2010). Patient characteristics and predictors of sleep medication use. Int. Clin. Psychopharmacol. 25, 91-100. doi: 10.1097/YIC.0b013e32833 $4 \mathrm{e} 5 \mathrm{e} 6$

Oosterloo, M., Lammers, G. J., Overeem, S., De Noord, I., and Kooij, J. J. (2006). Possible confusion between primary hypersomnia and adult attention-deficit/hyperactivity disorder. Psychiatry Res. 143, 293-297. doi: 10.1016/j.psychres.2006.02.009

Philipsen, A., Feige, B., Hesslinger, B., Ebert, D., Carl, C., Hornyak, M., et al. (2005). Sleep in adults with attention-deficit/hyperactivity disorder: a controlled polysomnographic study including spectral analysis of the sleep EEG. Sleep 28 , 877-884. doi: 10.1093/sleep/28.7.877

Philipsen, A., Hornyak, M., and Riemann, D. (2006). Sleep and sleep disorders in adults with attention deficit/hyperactivity disorder. Sleep Med. Rev. 10, 399-405. doi: 10.1016/j.smrv.2006.05.002

Roth, T., Zammit, G., Kushida, C., Doghramji, K., Mathias, S. D., Wong, J. M., et al. (2002). A new questionnaire to detect sleep disorders. Sleep Med. 3, 99-108. doi: 10.1016/S1389-9457(01)00131-9

Schredl, M., Alm, B., and Sobanski, E. (2007). Sleep quality in adult patients with attention deficit hyperactivity disorder (ADHD). Eur. Arch. Psychiatry Clin. Neurosci. 257, 164-168. doi: 10.1007/s00406-006-0703-1

Silvestri, R., Gagliano, A., Arico, I., Calarese, T., Cedro, C., Bruni, O., et al. (2009). Sleep disorders in children with Attention-Deficit/Hyperactivity Disorder (ADHD) recorded overnight by video-polysomnography. Sleep Med. 10, 1132-1138. doi: 10.1016/j.sleep.2009.04.003

Sobanski, E., Schredl, M., Kettler, N., and Alm, B. (2008). Sleep in adults with attention deficit hyperactivity disorder (ADHD) before and during treatment with methylphenidate: a controlled polysomnographic study. Sleep 31, 375-381. doi: 10.1093/sleep/31.3.375

Surman, C. B., Adamson, J. J., Petty, C., Biederman, J., Kenealy, D. C., Levine, M., et al. (2009). Association between attention-deficit/hyperactivity disorder and sleep impairment in adulthood: evidence from a large controlled study. J. Clin. Psychiatry 70, 1523-1529. doi: 10.4088/JCP.08m 04514

Surman, C. B., and Roth, T. (2011). Impact of stimulant pharmacotherapy on sleep quality: post hoc analyses of 2 large, double-blind, randomized, placebo-controlled trials. J. Clin. Psychiatry 72, 903-908. doi: 10.4088/JCP.11m 06838

Surman, C. B., Thomas, R. J., Aleardi, M., Pagano, C., and Biederman, J. (2006). Adults with ADHD and sleep complaints: a pilot study identifying sleep-disordered breathing using polysomnography and sleep quality assessment. J. Atten. Disord. 9, 550-555. doi: 10.1177/10870547052 82121

Tobaldini, E., Costantino, G., Solbiati, M., Cogliati, C., Kara, T., Nobili, L., et al. (2017). Sleep, sleep deprivation, autonomic nervous system and cardiovascular diseases. Neurosci. Biobehav. Rev. 74, 321-329. doi: 10.1016/j.neubiorev.2016.07.004

Van Veen, M. M., Kooij, J. J., Boonstra, A. M., Gordijn, M. C., and Van Someren, E. J. (2010). Delayed circadian rhythm in adults with attention-deficit/hyperactivity disorder and chronic sleep-onset insomnia. Biol. Psychiatry 67, 1091-1096. doi: 10.1016/j.biopsych.2009.12.032

Wagner, M. L., Walters, A. S., and Fisher, B. C. (2004). Symptoms of attentiondeficit/hyperactivity disorder in adults with restless legs syndrome. Sleep 27 1499-1504. doi: 10.1093/sleep/27.8.1499

Wilens, T. E., Biederman, J., Faraone, S. V., Martelon, M., Westerberg, D., and Spencer, T. J. (2009). Presenting ADHD symptoms, subtypes, and comorbid 
disorders in clinically referred adults with ADHD. J. Clin. Psychiatry 70, 1557-1562. doi: 10.4088/JCP.08m04785pur

Yoon, S. Y., Jain, U. R., and Shapiro, C. M. (2013). Sleep and daytime function in adults with attention-deficit/hyperactivity disorder: subtype differences. Sleep Med. 14, 648-655. doi: 10.1016/j.sleep.2013.03.003

Yoon, S. Y., Jain, U., and Shapiro, C. (2012). Sleep in attention-deficit/hyperactivity disorder in children and adults: past, present, and future. Sleep Med. Rev. 16, 371-388. doi: 10.1016/j.smrv.2011.07.001

Youssef, N. A., Ege, M., Angly, S. S., Strauss, J. L., and Marx, C. E. (2011). Is obstructive sleep apnea associated with ADHD? Ann. Clin. Psychiatry 23, 213-224.

Zak, R., Fisher, B., Couvadelli, B. V., Moss, N. M., and Walters, A. S. (2009). Preliminary study of the prevalence of restless legs syndrome in adults with attention deficit hyperactivity disorder. Percept. Mot. Skills 108, 759-763. doi: $10.2466 /$ pms.108.3.759-763
Conflict of Interest Statement: JH has received lecture honoraria as part of continuing medical education programs sponsored by Novartis, Eli Lilly and Company, and Janssen-Cilag.

The other authors declare that the research was conducted in the absence of any commercial or financial relationships that could be construed as a potential conflict of interest.

Copyright (๑ 2017 Bjorvatn, Brevik, Lundervold, Halmøy, Posserud, Instanes and Haavik. This is an open-access article distributed under the terms of the Creative Commons Attribution License (CC BY). The use, distribution or reproduction in other forums is permitted, provided the original author(s) or licensor are credited and that the original publication in this journal is cited, in accordance with accepted academic practice. No use, distribution or reproduction is permitted which does not comply with these terms. 


\section{APPENDIX}

Sleep questionnaire.

Have you ever had sleep problems lasting 1 month or longer?

$\square$ Yes $\square \mathrm{No}$

Have you ever been prescribed sleep medications?

$\square$ Yes $\square$ No

How will you rate your sleep? ${ }^{1}$

$\square$ very good $\square$ good $\square$ neither good nor bad $\square$ bad $\square$ verybad

How much sleep do you on average get per day? ___ hours ___ minutes

Are you a morning- or evening person?

$\square$ definite morning type $\square$ moderate morning type $\quad \square$ intermediate type $\square$ moderate evening type $\square$ definite evening type

During the past 4 weeks, how often did you fall asleep unintentionally or had to fight to stay awake during the day? ${ }^{2}$

$\square$ never $\square$ sometimes $\square$ usually $\square$ always

During the past 4 weeks, how often did you experience sudden loss of muscle tone (e.g. knees unlocking) by emotional reactions like laughter, anger or fear? ${ }^{3}$

$\square$ never $\square$ sometimes $\square$ usually $\square$ always

During the past 4 weeks, do you know (possibly through others) how often you have snored loudly?

$\square$ never $\square$ sometimes $\square$ usually $\square$ always

During the past 4 weeks, do you know (possibly through others) how often you have had breathing pauses or stopped breathing in your sleep?

$\square$ never $\square$ sometimes $\square$ usually $\square$ always

During the past 4 weeks, how often did you experience restless or crawling feelings in your legs in the evening or at night that went away if you moved your legs? ${ }^{4}$

$\square$ never $\square$ sometimes $\square$ usually $\square$ always

During the past 4 weeks, do you know (possibly through others) how often you have had repeated rhythmic leg jerks or leg twitches during your sleep ${ }^{5}$

$\square$ never $\square$ sometimes $\square$ usually $\square$ always

${ }^{1}$ Proxy for sleep quality.

${ }^{2}$ Proxy for excessive daytime sleepiness.

${ }^{3}$ Proxy for cataplexy (sign of narcolepsy).

${ }^{4}$ Proxy for restless legs.

${ }^{5}$ Proxy for periodic limb movements in sleep. 